\title{
Assessment of the Molecular Weight Distribution of Tannin Fractions through MALDI-TOF MS Analysis of Protein-Tannin Complexes
}

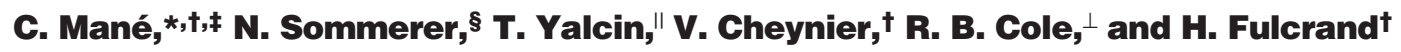

INRA, INRA,UMR1803 Sciences pour l'Oenologie, and UR1199 Laboratoire de Proteomique, F34060 Montpellier cedex, France, Comite Interprofessionnel des Vins de Champagne, 51204 Epernay cedex, France, Department of Chemistry, Izmir Institute of Technology, Urla-Izmir 35430, Turkey, and Department of Chemistry, University of New Orleans, New Orleans, Louisiana

An innovative mass spectrometry method was developed for determining mass distributions of tannin fractions that cannot be approached through direct MALDI-TOF analysis. It was applied to three procyanidin fractions with average degrees of polymerizations $=3,9$, and 28 , respectively, and one gallotannin fraction (Tara tannin). The proposed approach consists of MALDI-TOF analysis of the soluble complexes formed between these tannin fractions and bovine serum albumin (BSA). Complexes were detected as an unresolved "hump" following the BSA signal, and spectra were mathematically processed to determine the parameters relative to the protein-tannin complexes, which are the number-average molecular weight $\left(M_{\mathrm{n}}\right)$, the weight-average molecular weight $\left(M_{\mathrm{w}}\right)$, and the polydispersity index (PI) for each tannin fraction. Regarding condensed tannins, results are consistent with those of the standard method (thiolysis followed by HPLC separation) for all tested fractions. The method was successfully applied to a hydrolyzable tannin fraction but no standard method is available for comparison.

Tannins are a heterogeneous group of polyphenols widely present in the plant kingdom as secondary metabolites. They occur in bark, wood, fruits, fruit pods, leaves, roots, and plant galls. Tannins are classified into two groups: the hydrolyzable tannins, composed of a polyol central core acylated by a variable number of gallic (Figure 1) or ellagic acid units, and the proanthocyanidins, also called condensed tannins, which are oligomers and polymers of flavanol units (Figure 2). The common characteristics of tannins are (i) the range of their molecular weights (usually between 500 and 20000 ), (ii) the presence of free phenolic groups within the various subunits of their structure, and (iii) their ability to bind proteins and thereby form soluble and insoluble tannin-protein complexes. A growing interest has been addressed to this class of compounds because of their biological, nutritional, and sensory

\footnotetext{
* To whom correspondence should be addressed: (phone) +33499612023 (fax) +33499612857; (e-mail) mane@ensam.inra.fr.

$\dagger$ INRA, UMR1083 Sciences pour l'Oenologie, INRA.

* Comité Interprofessionnel des Vins de Champagne.

§ INRA,UR1199 Laboratoire de Protéomique.

"Izmir Institute of Technology.

${ }^{\perp}$ University of New Orleans.
}

properties. ${ }^{1-5}$ Regarding condensed tannins, their size (i.e., degree of polymerization) influences many of these properties, specifically astringency, which is a tactile sensation elicited by the precipitation of salivary proteins as a result of their binding to tannins. Recent works have actually demonstrated the increase in astringency perception with the increase in the average degree of polymerization (aDP) of the tannin fraction tasted. ${ }^{6}$ Determination of the $\mathrm{aDP}$ is quite easily achieved by standard methods referred to as thiolysis or phloroglucinolysis. ${ }^{7,8}$ These methods, based on acidcatalyzed cleavage of the interflavan linkages in the presence of a nucleophilic agent followed by HPLC analysis of the reaction products, give access to the nature and proportions of the different constitutive units within a condensed tannin fraction and allow calculation of its average degree of polymerization. However, they cannot provide the molecular weight distribution of the tannin fraction since all its constitutive polymers are cleaved. Analysis of hydrolyzable tannins is usually achieved by HPLC quantification of the gallic or ellagic acid units released after mild alkaline hydrolysis, but this gives access neither to the average number of gallic acids per molecule $(\mathrm{aDG})$ nor to the molecular weight distribution. Gel-permeation chromatography (GPC), also called size-exclusion chromatography (SEC), is the most widely used technique for the analysis of polymers because it yields the molecular weight distribution in addition to the molecular weight averages. The molecular weight and the range of molecular weights are classically determined from a calibration plot. SEC has been applied to both condensed ${ }^{8}$ and hydrolyzable ${ }^{9}$ tannins. In the case of condensed tannins, the results are quite consistent

(1) Haslam, E.; Lilley, T. H. Crit. Rev. Food Sci. Nutr. 1988, 27, 1-40.

(2) Cao, G.; Russell, R. M.; Lischner, N.; Prior, R. L. J. Nutr. 1998, 128, 23832390.

(3) Young, J. F.; Nielsen, S. E.; Haraldsdottir, J.; Daneshvar, B.; Lauridsen, S. T.; Knuthsen, P.; Crozie, A.; Sandstrom, B.; Dragsted, L. O. Am J. Clin. Nutr. 1999, 69, 87-94.

(4) Rein, D.; Lotito, S.; Holt, R. R.; Keen, C. L.; Schmitz, H. H.; Fraga, C. G. J. Nutr. 2000, 130, 2109S-2114S.

(5) Koga, T.; Meydani, M. Am. J. Clin. Nutr. 2001, 73, 941-948.

(6) Vidal, S.; Francis, L.; Guyot, S.; Marnet, N.; Kwiatkowski, M.; Gawel, R.; Cheynier, V.; Waters, E. J. J. Sci. Food Agric. 2003, 83, 564-573.

(7) Rigaud, J.; Perez-Ilzarbe, J.; Ricardo-da-Silva, J. M.; Cheynier, V. J. Chromatogr. 1991, 540, 401-405.

(8) Kennedy, J. A.; Taylor, A. W. J. Chromatogr. A 2003, 995, 99-107.

(9) Viriot, C.; Scalbert, A.; Hervé-du-Penhoat, C.; Rolando, C.; Moutounet, M. J. Chromatogr. A 1994, 662, 75-85. 


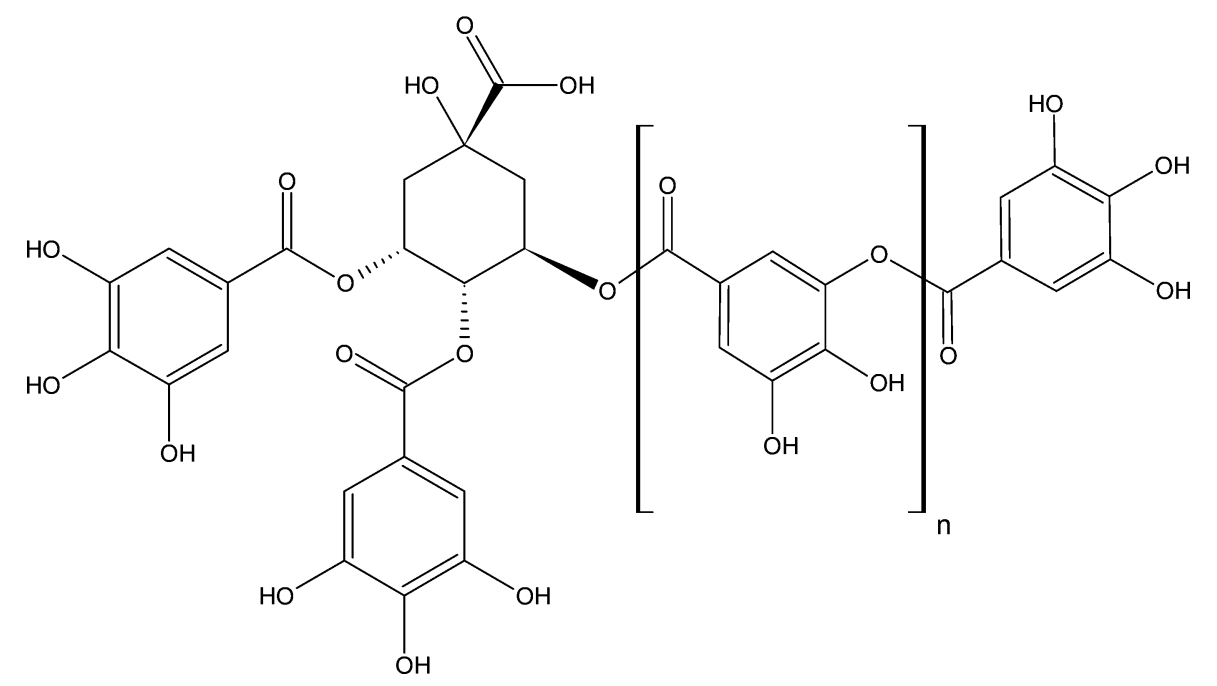

Figure 1. Tara tannin structure.

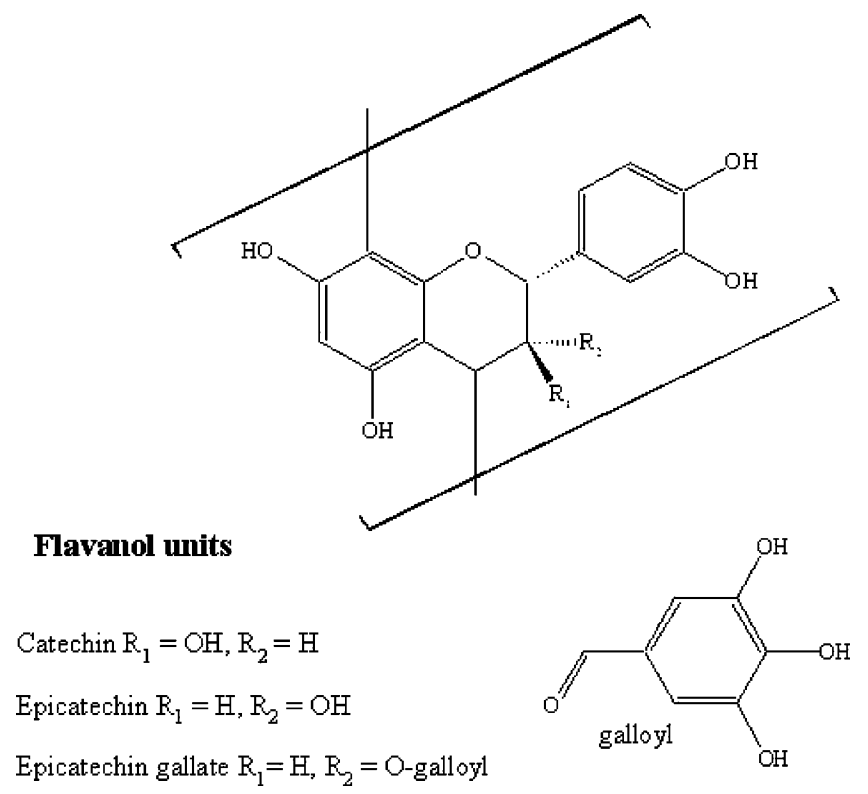

Figure 2. Structure of condensed tannins.

at low molecular weights, but they become inconsistent as the degree of polymerization increases. ${ }^{10}$ This may be due to the nature of the calibrants, as the polystyrene polymers used for this purpose are chemically and conformationally too different from the studied polyphenols. Therefore, GPC is primarily used as a separation technique for tannins rather than as a method to determine their molecular weight distribution, for which mass spectrometry could be an alternative approach.

Electrospray ionization (ESI) mass spectrometry has proven to be very efficient for structural analysis of polyphenols, but it has shown limitations in the evaluation of the molecular weight distributions of tannin mixtures. That is, ESI mass spectra of tannin mixtures are always dominated by the lowest molecular weight (MW) components with peak intensities diminishing as polymer chain length increases, in a pattern resembling exponential decay. ${ }^{11,12}$ This is also observed with matrix-assisted laser desorption ionization time-of-flight (MALDI-TOF) mass spectrom-

(10) Nonier, M. F.; Absalon, C.; Vivas, N.; Vivas-de-Gaulejac, N. J. Chromatogr. A 2004, 1033, 291-297. etry, although the latter has demonstrated a better ability to detect larger polymers than the ESI systems. The fractionation of crude tannin extracts prior to mass analysis greatly improves the detection of larger molecular weight species. ${ }^{11,12}$

Thus, the aim of the work presented here is to develop an original methodology to determine the molecular weight distribution of various tannin fractions through the detection of tanninprotein complexes by MALDI-TOF mass spectrometry. Indeed, the main characteristic of tannins is their ability to bind proteins, and protein-tannin complexes have already been observed by electrospray ${ }^{13,14}$ and MALDI-TOF ${ }^{15}$ mass spectrometry. The proposed approach relies upon the use of a protein to serve as a charge carrier for tannin detection as well as serving as a protective ligand against fragmentation, so as to minimize discrimination in ionization and reduce fragmentation of large tannin polymers. The MALDI-TOF technique has been selected because it yields mainly singly charged ions. Interpretation of tanninprotein complex spectra is thus easier than that of ESI mass spectra, for which superimposition of the protein charge-state distribution and tannin molecular weight distributions would lead to very complicated profiles of protein-tannin complexes. In addition to this, the MALDI technique does not require polymer standards for mass calibration.

\section{EXPERIMENTAL SECTION}

Reagents and Samples. Bovine serum albumin (BSA) (fraction V, fatty acid free, MW $\sim 66700$ ), $\beta$-casein (MW $\sim 24000$ ), equine myoglobin (MW 17000 ), and bovine $\beta$-lactoglobulin (MW $\sim 18400$ ), as well as trans-3,5-dimethoxy-4-hydroxycinnamic acid 98\%, $\alpha$-cyano-4-hydroxycinnamic acid, dihydroxybenzoic acid (DHB), trans-indoleacetic acid (t-IAA), and 3,5-dimethoxy-4hydroxycinnamic acid were purchased from Sigma Aldrich Co. (St. Louis, MO).

(11) Yanagida, A.; Kanda, T.; Shoji, T.; Ohnishi-Kameyama, M.; Nagata, T. J. Chromatogr. 1999, 855, 181-190.

(12) Taylor, A. W.; Barofsky, E.; Kennedy, J. A.; Deinzer, M. L. J. Agric. Food Chem. 2003, 51, 4101-4110.

(13) Sarni-Manchado, P.; Cheynier, V. J. Mass Spectrom. 2002, 37, 609-616.

(14) Vergé, S.; Richard, T.; Moreau, S.; Richelme-David, S.; Vercauteren, J.; Promé, J. C.; Monti, J. P. Tetrahedron Lett. 2002, 43, 2363-2366.

(15) Chen, Y.; Hagerman, A. E. J. Agric. Food Chem. 2004, 52, 4008-4011. 

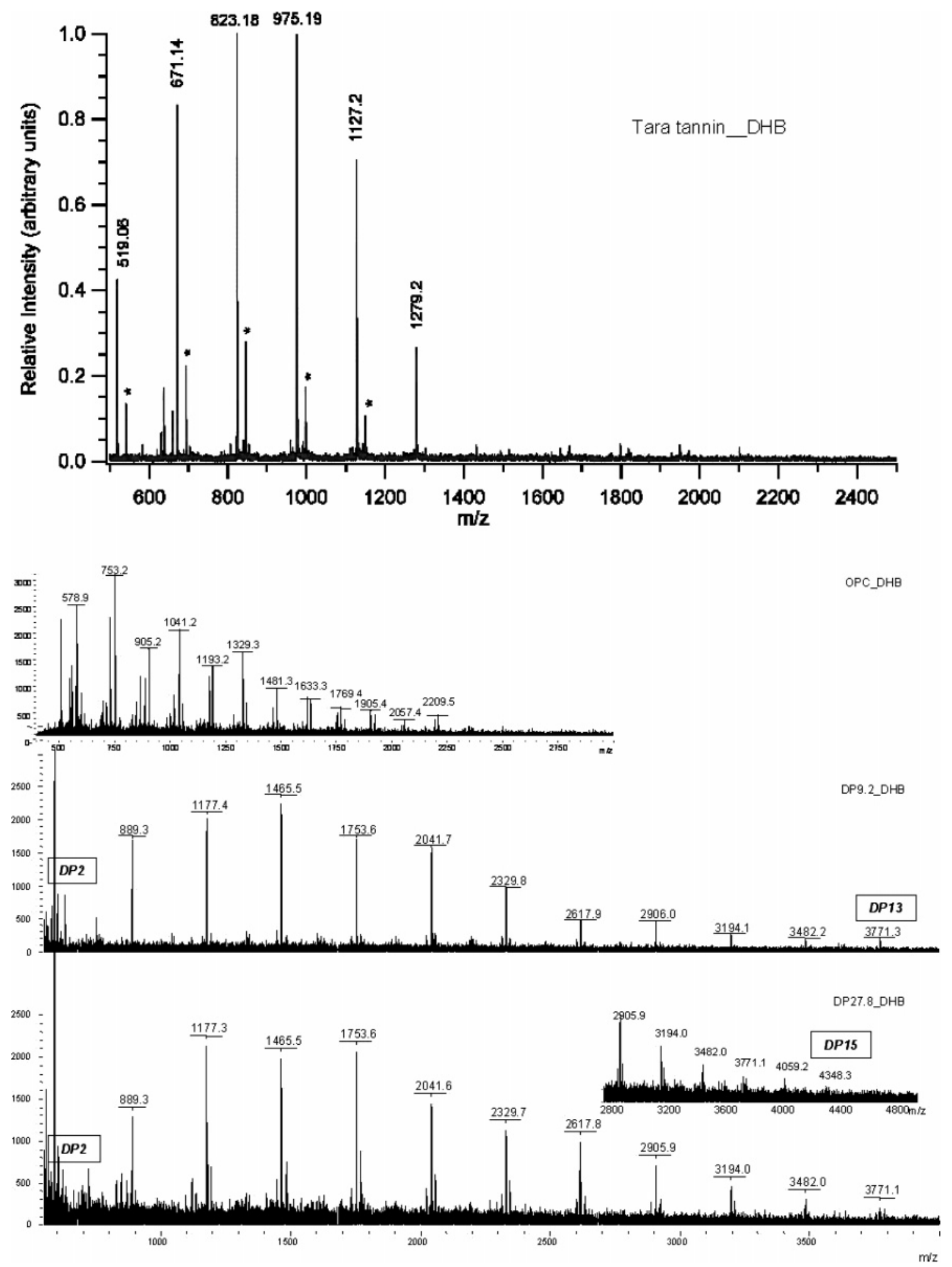

Figure 3. MALDI-TOF spectra of Tara tannin and condensed tannins (peak attribution is given in Tables 1 and 2).

Tara tannin, which is a mixture of gallotannins with quinic acid as the central core acylated by a variable number of gallic acid units (Figure 1), was purchased from Tara Mallinckrodt (Phillipsburg, NJ).

Oligomeric procyanidin fraction from grape seed (OPC) was purchased from Berkem (Gardonne, France). Thiolysis analysis of this fraction showed that it contained $17.5 \%$ galloylated units and its aDP was 2.6. Two condensed tannin fractions from apple
( $\mathrm{aDP}=9.2$ and 27.8, respectively, estimated by thiolysis) were kindly provided by Sylvain Guyot (Unité de Recherches Cidricoles, INRA, Rennes, France). As the nominal mass of a monomer is $290 \mathrm{Da}$, the average molecular weight (aMW) of each apple tannin fraction is given by the formula:

$$
\mathrm{aMW}=\mathrm{aDP}(288)+2
$$


Table 1. Composition of Tara Tannin Estimated from MALDI-TOF Spectrum of the Fraction

\begin{tabular}{cccc} 
Tara tannin $^{a}$ & MW & $\begin{array}{c}{[\mathrm{M}+\mathrm{Na}]^{+}} \\
\text {calculated }\end{array}$ & $\begin{array}{c}{[\mathrm{M}+\mathrm{Na}]^{+} m / z} \\
\text { detected }\end{array}$ \\
$1 \mathrm{G}$ & 344.07 & 367.06 & \\
$2 \mathrm{G}$ & 496.08 & 519.08 & 519.06 \\
$3 \mathrm{G}$ & 648.101 & 671.09 & 671.14 \\
$4 \mathrm{G}$ & 800.11 & 823.1 & 823.18 \\
$5 \mathrm{G}$ & 952.12 & 975.11 & 975.19 \\
$6 \mathrm{G}$ & 1104.13 & 1127.12 & 1127.2 \\
$7 \mathrm{G}$ & 1256.14 & 1279.13 & 1279.2 \\
$8 \mathrm{G}$ & 1408.15 & 1431.14 & 1431.2 \\
${ }^{a} \mathrm{G}=$ galloyl moiety. & & \\
\hline
\end{tabular}

That of the OPC fraction was calculated in the same way, but the proportion of galloylated units $(\% \mathrm{G})$, nominal mass $442 \mathrm{Da}$, was taken into account as follows:

$$
\mathrm{aMW}=\mathrm{aDP}[288(100-\% \mathrm{G}) / 100+440(\% \mathrm{G}) / 100)+2
$$

Acetic acid was purchased from VWR Prolabo (Fontenay-sousBois, France), and Centricon Ultracel YM-10 regenerated cellulose membrane was from Millipore (Bedford, MA).

Protein-Tannin Complexation. Solutions of protein (2 and $20 \mathrm{mg} / \mathrm{mL} ; 30$ and $300 \mu \mathrm{M}$, respectively) were prepared in $1 \%$ aqueous acetic acid, as was a $9 \mathrm{mM}$ solution of tannin. The molecular weight taken for calculating the molar concentration of condensed tannins was the average molecular weight determined by thiolysis. For hydrolyzable tannins, it was estimated by direct MALDI-TOF analysis. The solutions of protein and tannin were mixed for $0.5 \mathrm{~h}$ in four protein-to-tannin ratios, 10:1, 1:1, 1:10, and 1:300. Mixtures 1:1, 1:10, and 1:300 were submitted to an additional concentration step via use of a
$10 \mathrm{kDa}$ Centricon membrane, by centrifugation at $5000 \mathrm{~g}$ at $5{ }^{\circ} \mathrm{C}$ for $55 \mathrm{~min}$.

Unless otherwise specified, all mixtures were diluted in order to adjust the protein concentration to about $1 \mathrm{mg} / \mathrm{mL}$ before MALDI-TOF analysis.

Experiments were performed in duplicate.

General Instrumentation and Conditions. Analyses were performed on a BiFlex III MALDI-TOF mass spectrometer (Bruker Daltonics, Bremen, Germany). The mass spectra were acquired in linear mode over a mass range of 60-120 kDa for analyses of protein-tannin complexes, and in reflectron mode for direct analyses of tannin fractions over a mass range of 500-5000 Da, by use of an adjusted accelerating voltage.

MALDI-TOF spectra of tannin fractions and protein-tannin complexes were run with DHB, t-IAA, trans-3,5-dimethoxy-4hydroxycinnamic acid 98\%, $\alpha$-cyano-4-hydroxycinnamic acid, and 3,5-dimethoxy-4-hydroxycinnamic acid $98 \%$ as matrixes.

The matrix solutions were prepared at a $10 \mathrm{mg} / \mathrm{mL}$ concentration in $50 \%$ aqueous $\mathrm{CH}_{3} \mathrm{CN}$, acidified with $0.1 \%$ trifluoroacetic acid (TFA), and premixed with the protein-tannin solutions $(50 \% \mathrm{v} / \mathrm{v})$ After brief mixing of the analyte and the matrix, $1 \mu \mathrm{L}$ of the mixture was added on the MALDI target and allowed to air-dry. Mass calibration was performed externally. In this study, 700 laser shots were summed to obtain the final spectrum.

Mathematical Processing of Data. The molecular weight distributions of the different tannin fractions were characterized by the classical polymer parameters as follows:

Number-average molecular weight:

$$
M_{\mathrm{n}}=\frac{\sum(m / z)_{i} I_{i}}{\sum I_{i}} \text { with } I=\text { absolute intensity }
$$

\begin{tabular}{|c|c|c|c|c|c|c|c|c|c|}
\hline \multirow[b]{2}{*}{$\begin{array}{l}\text { condensed } \\
\text { tannins }\end{array}$} & \multirow[b]{2}{*}{ MW } & \multirow[b]{2}{*}{$\begin{array}{l}{[\mathrm{M}+\mathrm{Na}]^{+}} \\
\quad \text { calcd }\end{array}$} & \multirow[b]{2}{*}{$\begin{array}{l}{[\mathrm{M}+\mathrm{K}]^{+}} \\
\quad \text { calcd }\end{array}$} & \multicolumn{2}{|c|}{$\mathrm{OPC}$} & \multicolumn{2}{|c|}{ DP 9.2} & \multicolumn{2}{|c|}{ DP 27.8} \\
\hline & & & & $\begin{array}{c}{\left[\mathrm{M}+\mathrm{Na}^{+} m / z\right.} \\
\text { detected }\end{array}$ & $\begin{array}{c}{[\mathrm{M}+\mathrm{K}]^{+} m / z} \\
\text { detected }\end{array}$ & $\begin{array}{c}{[\mathrm{M}+\mathrm{Na}]^{+} m / z} \\
\text { detected }\end{array}$ & $\begin{array}{c}{[\mathrm{M}+\mathrm{K}]^{+} m / z} \\
\text { detected }\end{array}$ & $\begin{array}{c}{[\mathrm{M}+\mathrm{Na}]^{+} m / z} \\
\text { detected }\end{array}$ & $\begin{array}{c}{[\mathrm{M}+\mathrm{K}]^{+} m / z} \\
\text { detected }\end{array}$ \\
\hline DP2 & 578.13 & 601.12 & 617.22 & - & + & + & - & - & - \\
\hline DP2 $1 \mathrm{G}$ & 730.14 & 753.13 & 769.23 & + & + & - & - & - & - \\
\hline DP3 & 866.2 & 889.19 & 905.29 & - & + & + & - & + & - \\
\hline DP3 $1 G$ & 1018.21 & 1041.2 & 1057.30 & + & + & - & - & - & - \\
\hline DP4 & 1154.26 & 1177.25 & 1193.35 & - & + & + & - & + & + \\
\hline $\mathrm{DP} 41 \mathrm{G}$ & 1306.27 & 1329.26 & 1345.36 & + & + & - & - & - & - \\
\hline $\mathrm{DP} 42 \mathrm{G}$ & 1458.28 & 1481.27 & 1497.37 & + & + & - & - & - & - \\
\hline DP5 & 1442.32 & 1465.31 & 1481.41 & - & + & + & - & + & + \\
\hline DP5 $1 G$ & 1594.34 & 1617.33 & 1633.43 & + & + & - & - & - & - \\
\hline DP5 $2 \mathrm{G}$ & 1746.35 & 1769.34 & 1785.44 & + & + & - & - & - & - \\
\hline DP6 & 1730.39 & 1753.38 & 1769.48 & - & + & + & + & + & + \\
\hline DP6 $1 G$ & 1882.4 & 1905.39 & 1921.49 & - & + & - & - & - & - \\
\hline DP6 2G & 2034.41 & 2057.4 & 2073.5 & + & + & - & - & - & - \\
\hline DP7 & 2018.45 & 2041.44 & 2057.54 & - & + & + & - & + & + \\
\hline DP7 $1 G$ & 2170.46 & 2193.45 & 2209.55 & - & + & - & - & - & - \\
\hline DP7 2G & 2322.47 & 2345.46 & 2361.56 & & & - & - & - & - \\
\hline DP8 & 2306.51 & 2329.5 & 2345.60 & - & - & + & - & + & + \\
\hline DP9 & 2594.58 & 2617.57 & 2633.67 & - & - & + & - & + & + \\
\hline DP10 & 2882.64 & 2905.63 & 2921.73 & - & - & + & - & + & + \\
\hline DP11 & 3170.7 & 3193.69 & 3209.79 & - & - & + & - & + & + \\
\hline DP12 & 3458.76 & 3481.75 & 3497.85 & - & - & + & - & + & + \\
\hline DP13 & 3746.82 & 3769.81 & 3785.91 & - & - & + & - & + & + \\
\hline DP14 & 4034.88 & 4057.87 & 4073.97 & - & - & - & - & + & - \\
\hline DP15 & 4322.94 & 4345.93 & 4362.03 & - & - & - & - & + & - \\
\hline
\end{tabular}

Table 2. Composition of Condensed Tannin Estimated from MALDI-TOF Spectra of the Fractions 
Weight-average molecular weight:

$$
M_{\mathrm{w}}=\frac{\sum(m / z){ }_{i}{ }_{i} I_{i}}{\sum(m / z){ }_{i} I_{i}}
$$

Polydispersity index:

$$
\mathrm{PI}=\frac{M_{\mathrm{w}}}{M_{\mathrm{n}}}
$$

\section{RESULTS AND DISCUSSION}

Direct Analysis of the Tannin Fractions by MALDI-TOF. A series of tannin fractions, both hydrolyzable and condensed, was chosen so as to cover a large range of average molecular weight values. Tara gallotannin was selected as the hydrolyzable tannin fraction. Direct MALDI-TOF analysis of this fraction showed an ion series within the mass range of 500-1500 (Figure 3 , top). The mass difference between two successive ion peaks was equal to 152 , readily assigned to an additional galloyl residue. The main series corresponds to monosodium adducts as shown by comparison with the expected molecular weights (Table 1 ). The lowest peak in the series obtained under our conditions was detected at $m / z=519.06$ and attributed to a quinic acid molecule substituted with two galloyl groups, while the highest one was detected at 1431.2 and attributed to a quinic acid substituted with eight galloyl units. Another ion series (designated by asterisks in the spectrum presented in Figure 3), accounting for approximatively $25 \%$ of the total signal, was detected at 22 units more than the former one, which corresponds to the singly charged disodium ion series. From this spectrum, the average number of galloyl units was estimated to be around $4-5$, which corresponds to the maximum as well as to the median of the galloyl distribution. The number-average molecular weight $\left(M_{\mathrm{n}}\right)$ of this tannin fraction calculated from the intensities of each peak was 860 .
For condensed tannins, three fractions differing by the nature of their constitutive units and their aDP values have been selected, namely, two apple proanthocyanidin fractions consisting of epicatechin polymers (aDP 9.2 and 27.8, respectively) and one grape seed OPC fraction characterized by the presence of a galloyl residue on some of the repeating (epi)catechin units $(\mathrm{aDP}=2.6$, $17.5 \%$ galloyl residues). The ion series in the OPC spectrum corresponds to the nongalloylated, monogalloylated, and digalloylated (epi)catechin oligomers ranging from dimer to heptamer (sodium or potassium adducts), characterized by a mass difference of 288 within the nongalloylated (epi)catechin oligomers and a mass difference of 152 within the corresponding galloylated species (Table 2). Note that the occurrence of both sodium and potassium adducts for the galloylated procyanidins complicates interpretation, as the sodium adduct of a $\mathrm{DP}_{n}$ molecule with two galloyl substituents overlaps with the potassium adduct of the nongalloylated $\mathrm{DP}_{n+1}$ species. The $M_{\mathrm{n}}$ value for this fraction was calculated from the intensities of peaks in the MALDI-TOF spectrum at 916 and is thus consistent with that estimated from thiolysis (820).

The ion series of both apple tannin fractions correspond to potassium and/or sodium adduct series of epicatechin oligomers with mass differences of $288 \mathrm{amu}$. However, no clear difference between these two fractions could be distinguished in terms of mass distribution (Figure 3). The spectrum of the aDP 9.2 fraction shows an oligomer series ranging from DP3 to DP13, while the spectrum of the aDP 27.8 fraction shows an ion series ranging from DP2 to DP15. The $M_{\mathrm{n}}$ values calculated for these apple tannin fractions were 1562 and 1908, respectively, instead of 2652 and 8008 , respectively, as calculated from the thiolysis data. This suggests that the ion peak intensity may be biased toward smaller sized oligomers. The lower intensities detected for large oligomers may result from preferential desorption/ionization and/or fragmentation of larger species (in both cases leading to an overestimation of lower DP); these problems are likely more pronounced

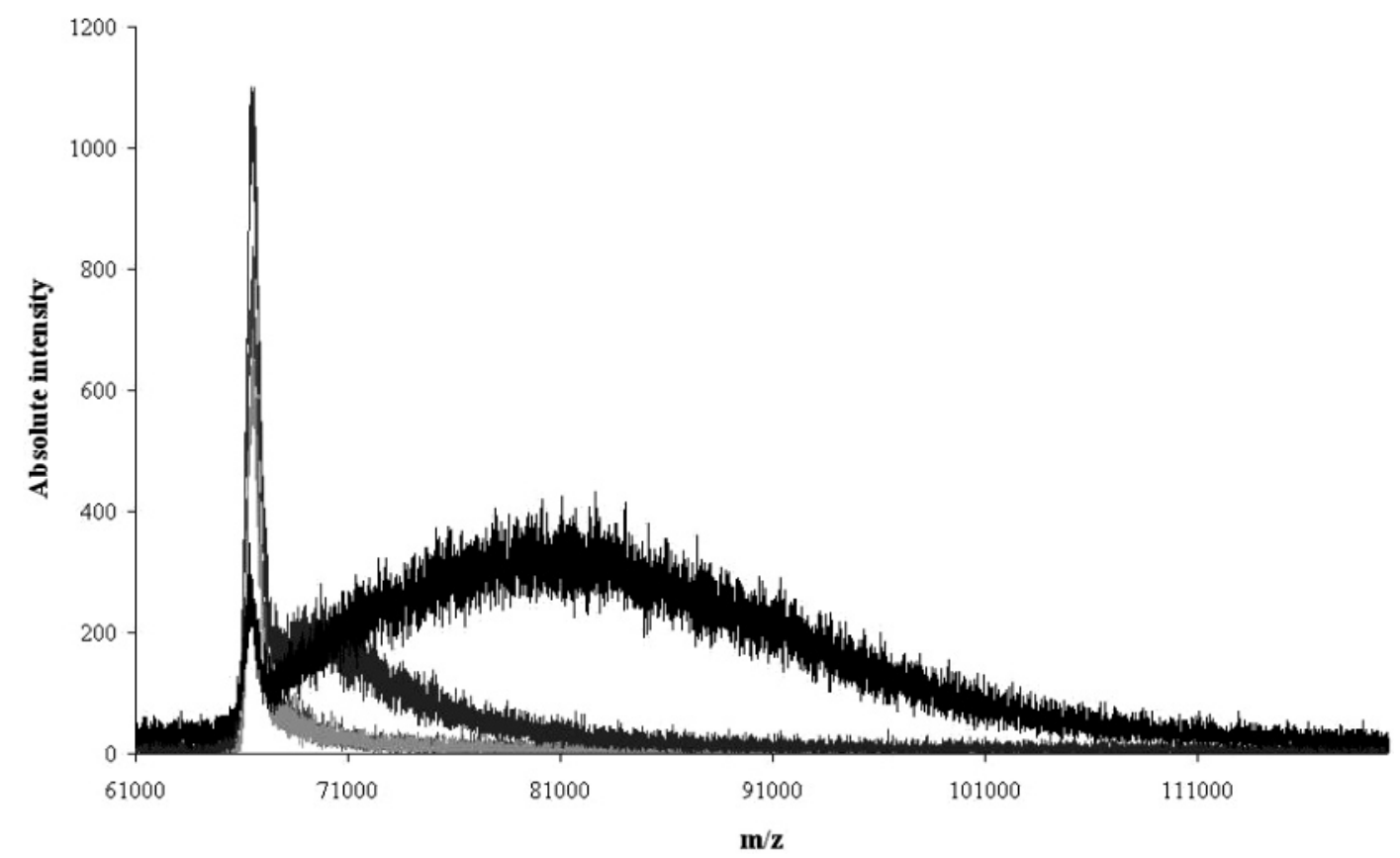

Figure 4. MALDI-TOF mass spectra of mixtures of BSA (medium gray), BSA-DP 9.2 10:1 (light gray), BSA-DP 9.2 1:1 (dark gray), and BSA-DP9.2 1:10 (black). 


\section{Table 3. Evaluation of aDP for Each Tannin Fraction by Observation of the Signals of Free BSA and Complexes}

\begin{tabular}{|c|c|c|c|c|c|c|c|c|}
\hline \multirow[b]{3}{*}{ Tara tannin } & \multicolumn{4}{|c|}{ direct analysis of fractions } & \multicolumn{4}{|c|}{ MALDI-TOF analysis of complexes ${ }^{a}$} \\
\hline & \multicolumn{2}{|c|}{ thiolysis } & \multicolumn{2}{|c|}{ MALDI-TOF } & \multicolumn{2}{|c|}{ mean mol wt } & \multicolumn{2}{|c|}{$\mathrm{aDP} / \mathrm{aDG}$} \\
\hline & av mol wt & $\mathrm{aDP} / \mathrm{aDG}$ & $\begin{array}{l}M_{\mathrm{n}} \\
860\end{array}$ & $\begin{array}{c}\mathrm{aDP} / \mathrm{aDG} \\
4.5\end{array}$ & $\begin{array}{c}\text { series } 1 \\
950\end{array}$ & $\begin{array}{c}\text { series } 2 \\
982\end{array}$ & $\begin{array}{c}\text { series } 1 \\
5.0\end{array}$ & $\begin{array}{c}\text { series } 2 \\
5.2\end{array}$ \\
\hline $\mathrm{OPC}$ & 820 & 2.6 & 916 & 2.9 & 1552 & 1531 & 4.9 & 4.8 \\
\hline DP 9.2 & 2652 & 9.2 & 1562 & 5.4 & 2672 & 2894 & 9.3 & 10.0 \\
\hline DP 27.8 & 8008 & 27.8 & 1908 & 6.6 & 3199 & 3157 & 11.1 & 11.1 \\
\hline
\end{tabular}

${ }^{a}$ Analysis of BSA-tannin complexes by the difference between the top of the "hump" and free BSA signals.

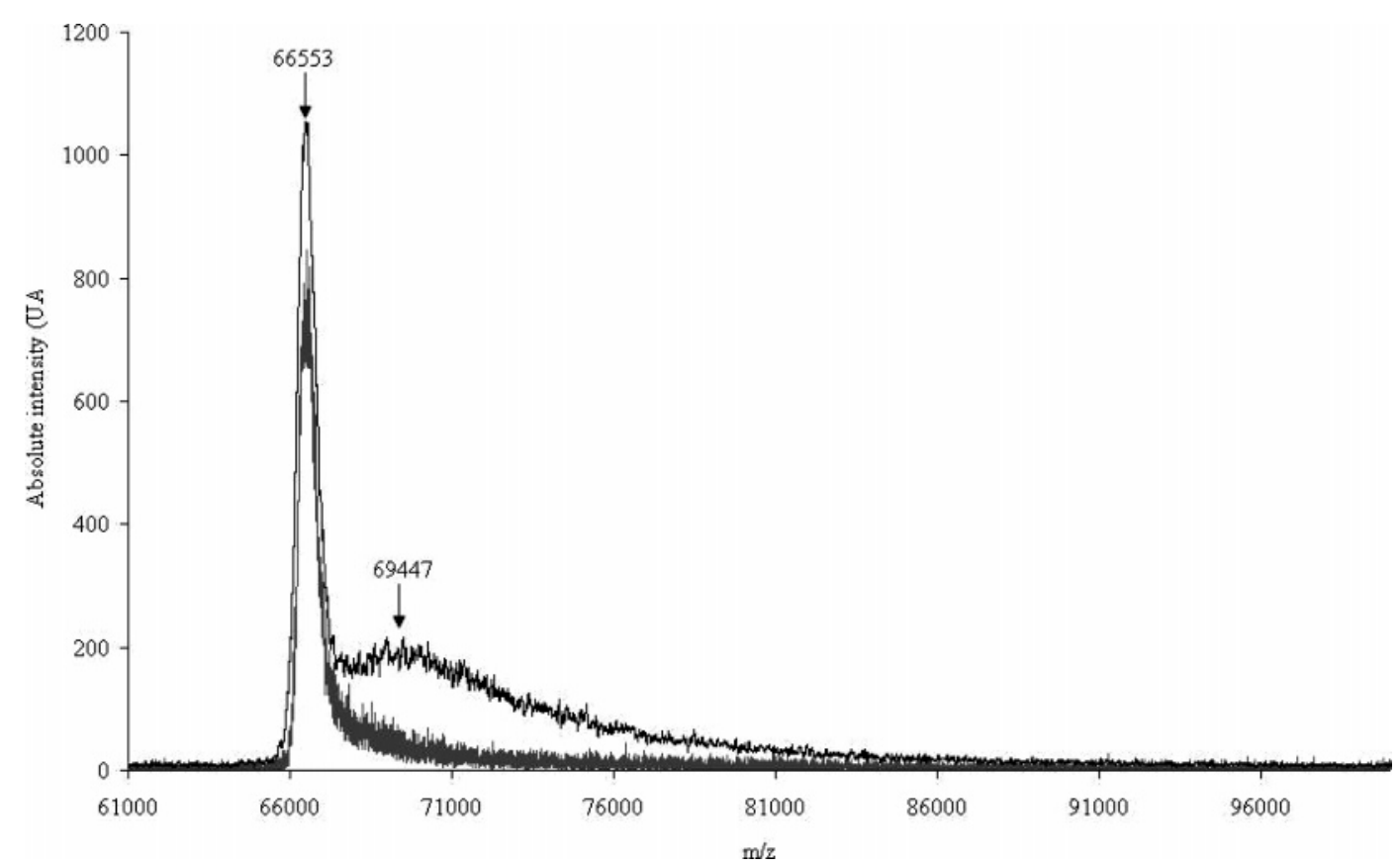

Figure 5. MALDI-TOF mass spectra in positive linear mode of BSA (black) and BSA in the presence of DP 9.2 (gray).

when a wide polydispersity exists for the tannin fractions. Our studies show that the discrepancy between the molecular weight distribution observed by mass spectrometry and the average molecular weight determined by thiolysis increases with the degree of polymerization. This is in agreement with earlier studies revealing that higher molecular weight tannins are not efficiently detected by MS, especially in highly polydisperse tannin mixtures, due at least in part to fragmentation. ${ }^{12}$

Direct analysis of tannin fractions by MALDI-TOF mass spectrometry thus appears useful to establish mass distributions of oligomeric procyanidins, but it likely is most suitable for the lower molecular weight tannins such as Tara gallotannins; however, this cannot be definitively established because of the lack of a reliable reference method. Clearly, direct analysis becomes less reliable as the molecular weight increases. Therefore, we sought to employ proteins as binding agents capable of protecting tannins against fragmentation while simultaneously serving as charge carriers to allow determination of the mass distributions of higher molecular weight tannins.

Analyses of Protein-Tannin Complexes by MALDI-TOF: Selection of the Protein Used as Charge Carrier. Tannin-protein interactions are influenced by properties of both the protein and the tannin. Protein characteristics that usually favor strong binding are large molecular size, plus open and flexible structure. ${ }^{16,17}$ The first step of our study aimed at selecting proteins that have strong affinities for tannin fractions and for which complexes are readily detected by mass spectrometry. Several proteins were tested in order to examine a large range of molecular weights because the requirements for strong binding and mass spectral quality seemed a priori in opposition (large molecular size for binding and impeding the fragmentation of larger tannins vs small size for good resolution). Availability and cost were also essential criteria for selecting the protein. Afterward, experimental optimization was performed by varying parameters such as the protein-to-tannin ratio and the dilution rate of mixtures and matrix. Bovine serum albumin, BSA, which is known to form complexes with tannins, ${ }^{18}$ was the first protein selected. Experiments were first carried out in the presence of a large excess of OPC, corresponding to a protein-to-tannin ratio of 1:300. After shaking for $30 \mathrm{~min}$, mixtures of tannin and protein solutions were centrifuged, thereby forming a solid pellet. The mixture of tannins and proteins in solution leads to the formation of both soluble and insoluble complexes within the pellet; their relative proportion depends on the concentration and size of both molecules. Soluble complexes are favored when

(16) Hagerman, A. E. In Chemistry and significance of condensed tannins; Hemingway, R. W., Karchesy, J. J., Eds.; Plenum Press: New York, London, 1989; pp 323-331.

(17) Charlton, A. J.; Baxter, N. J.; Lilley, T. H.; Haslam, E.; McDonald, C. J.; Williamson, M. P. FEBS Lett. 1996, 382, 289-292.

(18) Hagerman, A. E.; Butler, L. G. J. Agric. Food Chem. 1978, 26, 809-812. 

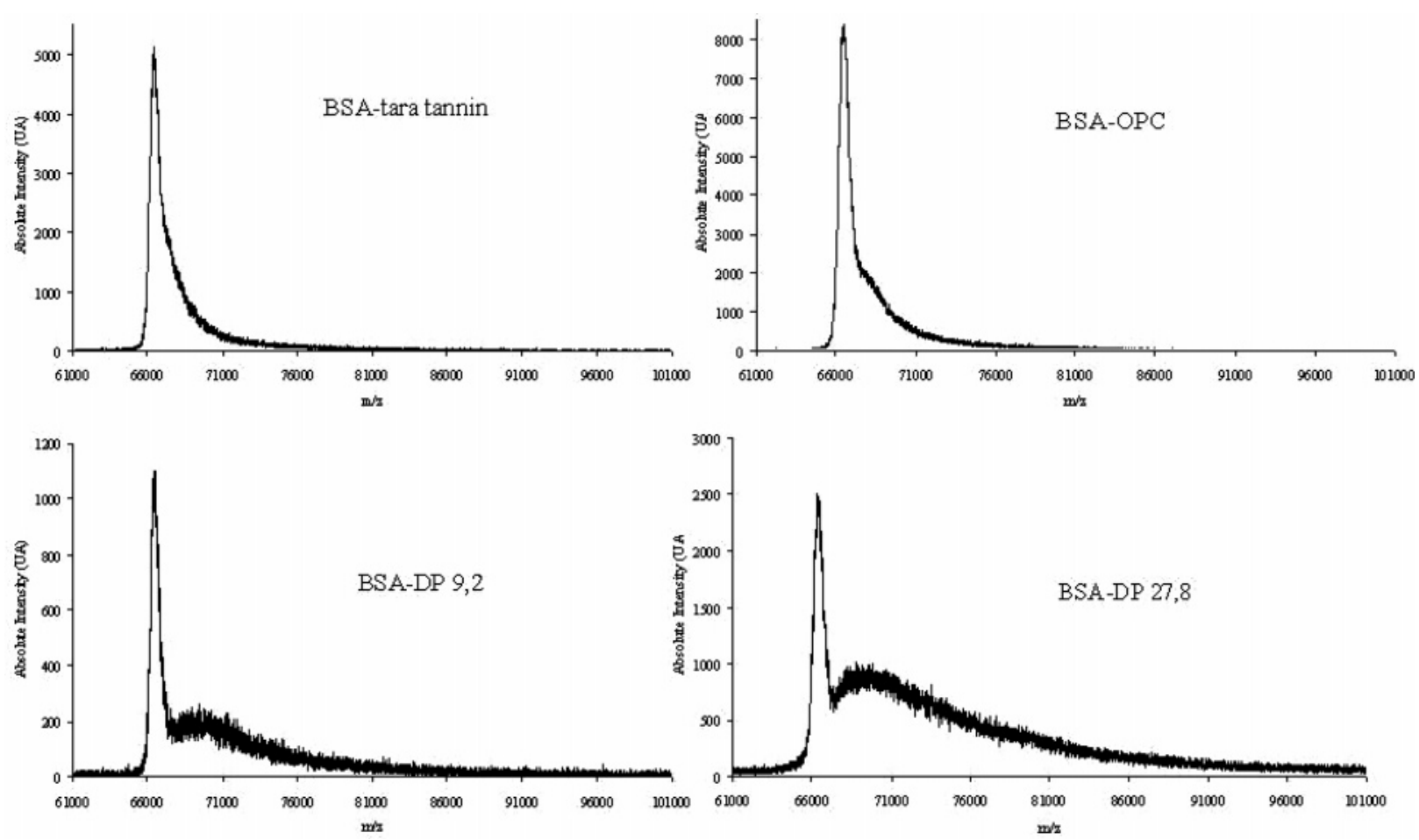

Figure 6. MALDI-TOF spectra of 1:1 complexes BSA-Tara tannin, BSA-OPC, BSA-DP 9.2, and BSA-DP 27.8.

protein concentration is in excess, whereas insoluble complexes are formed when tannins are present in excess. ${ }^{19}$ Soluble complexes represent an analytical problem because they are differentiated neither by UV-visible spectrophotometry nor by dynamic light scattering from free (unbound) tannins. For our purpose, soluble complexes can be directly analyzed by MALDI-TOF MS. Supernatants were withdrawn and filtered through a $10 \mathrm{kDa}$ Centricon filter. The retentate (corresponding to the protein fraction that contains free protein and soluble tannin-protein complexes) was added to the HCCA matrix solution. Its analysis showed a large unresolved "hump" that starts at $m / z$ values above the BSA signal and may correspond to the molecular weight distribution of the OPC-BSA complexes. No discrete $m / z$ values corresponding to OPC-BSA complexes can be distinguished due to the lack of resolution in this mass range. Analyses of the OPCBSA pellets first dissolved in $30 \%$ aqueous dimethyl sulfoxide (DMSO) showed mass profiles similar to that obtained with the soluble fraction and thus did not bring additional information. Moreover, the use of DMSO is not optimal for the sample preparation, as it slows the drying of the deposit on the target.

Three other proteins, namely, $\beta$-casein (used for wine fining), equine myoglobin, and bovine $\beta$-lactoglobulin, known to bind to various hydrophobic molecules were also tested. Complexes resulting from interactions between OPC and $\beta$-lactoglobulin, $\beta$-casein, or equine myoglobin were not detected by MALDI-TOF MS. However, OPC $-\beta$-casein complexes could be visualized by ESI MS (data not shown).

The spotting method was also evaluated using different matrixes along with different optimization steps for BSA-OPC complexes. $\alpha$-Cyano-4-hydroxycinnamic acid, t-IAA, and 3,5dimethoxy-4-hydroxycinnamic acid were tested with double-

(19) McManus, J. P.; Davis, K. G.; Beart, J. E.; Galffney, S. H.; Lilley, T. H.; Haslam, E. J. Chem. Soc., Perkin Trans. 2 1985, 1429-1438. droplet or thin-layer spotting, and an additional assay was carried out by a two-layer matrix method (first layer of 3,5-dimethoxy-4hydroxycinnamic acid in methanol/acetone, second layer of sinapic acid in methanol/water). The best signal-to-noise mass spectrum was obtained with $\alpha$-cyano-4-hydroxycinnamic acid as matrix with double-droplet spotting.

The same protocol was repeated except that OPC was replaced by aDP 9.2 apple tannin fraction. The MALDI-TOF mass spectra of both supernatant and pellet showed again a similar hump after the BSA signal. However, no significant difference could be detected in the shape of the unresolved hump obtained with BSAOPC and BSA-apple tannin solutions, contrary to what might be expected from their different aDP values. This observation can be explained by the large excess of polyphenols in the mixtures for which may saturate of all the binding sites available on BSA for both tannin fractions.

Optimization of the Protein-to-Tannin Ratio. To refine the ability to use proteins in evaluating tannin distributions, it was thus necessary to adjust the protein-to-tannin ratio in order to detect complexes with lower stoichiometries, and particularly to favor those with one tannin molecule per protein molecule (1:1 stoichiometry). Therefore, 10:1, 1:1, and 1:10 protein-to-tannin molar ratios were prepared with the apple tannin fraction. The first consequence of increasing the protein-to-tannin ratio was to keep all complexes soluble regardless of the ratio, which led to a higher overall concentration of complexes in solution than that obtained with the 1:300 molar ratio. Additionally, several dilution levels of the spotted mixtures were tested. The best spectrum was obtained by lowering the BSA concentration to $1 \mathrm{mg} / \mathrm{mL}$ prior to analysis and this condition was kept afterward. Moreover, trans-3,5dimethoxyhydroxycinnamic acid was the most appropriate matrix with these new ratios. Figure 4 shows the comparison of the spectra obtained at different molar ratios. First, the MALDI- 


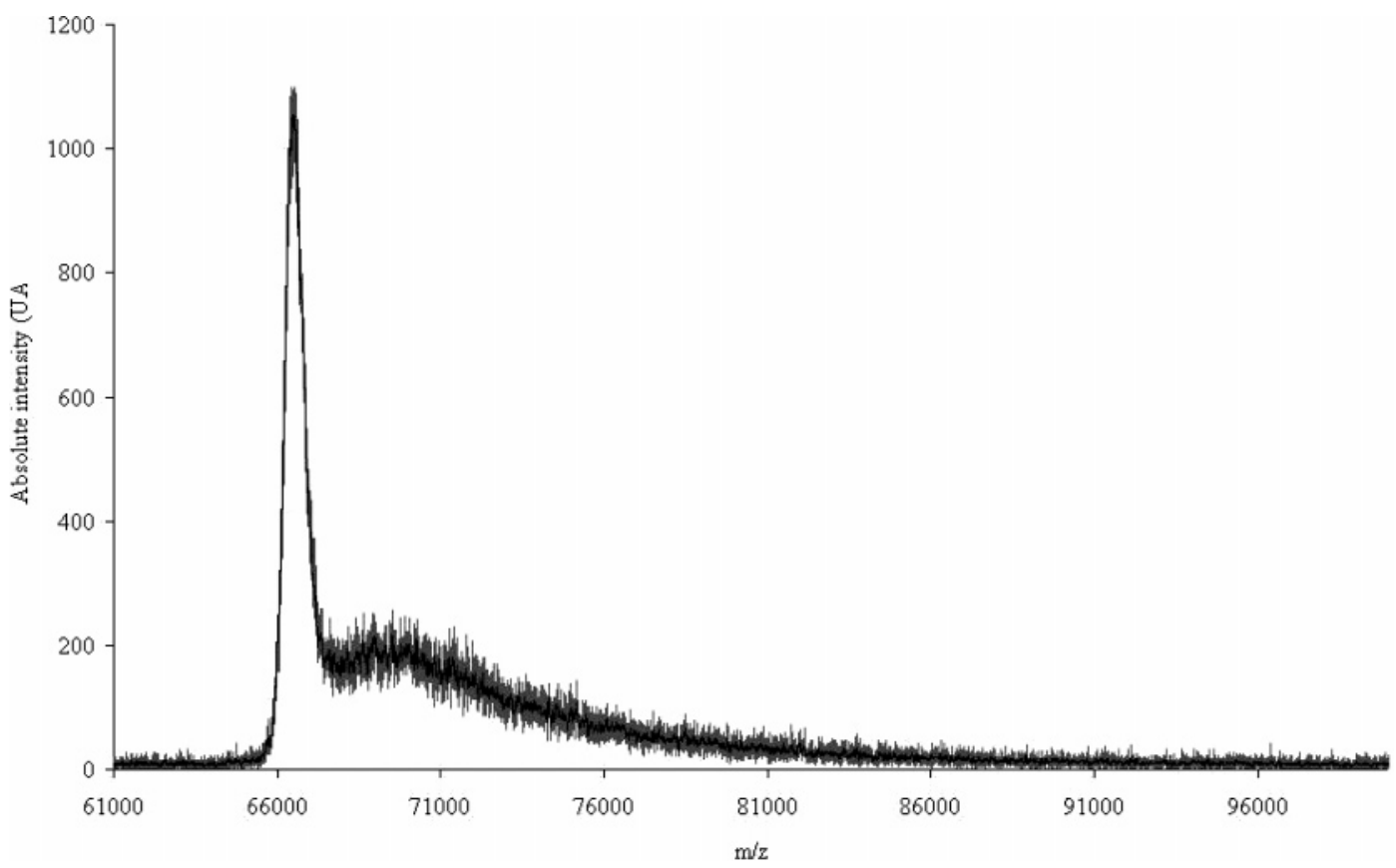

Figure 7. Raw spectrum (gray) and processed curve (black) of BSA-DP 9.2.

Table 4. Number- and Weight-Average Molecular Weights of BSA and BSA-Tannin Complexes

\begin{tabular}{lccccc} 
& \multicolumn{2}{c}{ series 1} & & \multicolumn{2}{c}{ series 2} \\
\cline { 2 - 3 } \cline { 6 - 6 } & $M_{\mathrm{n}}$ & $M_{\mathrm{w}}$ & & $M_{\mathrm{n}}$ & $M_{\mathrm{w}}$ \\
BSA & 66946 & 66961 & & 67197 & 67218 \\
BSA-Tara tannin & 67870 & 67935 & & 68235 & 68378 \\
BSA-OPC & 67961 & 68095 & & 68358 & 68515 \\
BSA-DP 9.2 & 69596 & 69759 & & 69985 & 70216 \\
BSA-DP 27.8 & 75010 & 76472 & & 75008 & 76304 \\
\hline
\end{tabular}

TOF mass spectra of BSA standard and 10:1 mixture are heavily overlapping, indicating that the $10: 1$ ratio was not appropriate. This does not imply that no complex is formed in the 10:1 mixture, but rather that they cannot be detected because of the lack of resolution. The mass spectra obtained from the other two ratios showed that increasing the apple tannin proportion results in the widening of the hump and the shift of its maximum toward higher $m / z$. In the case of the 1:1 mixture, the mass difference between the maximum of the hump and the maximum of the BSA signal amounts to $2672 \mathrm{Da}$ and is thus consistent with the value estimated by thiolysis (2652 Da) as given in Table 3 .

This congruence indicates that complexes with a 1:1 stoichiometry are the major population appearing along with the free BSA and, thus, that the hump reflects the complex distribution of the tannin fraction (Figure 5). On the other hand, the shift of the hump toward higher molecular weights with the 1:10 mixture (tannin excess) suggests the existence of complexes with much higher stoichiometries. Picking up the maximum of the hump at 81000 leads to an estimated average stoichiometry of five tannin molecules per protein molecule. Further experiments held the protein-to-tannin molar ratio at $1: 1$ because, under these conditions, the mass signal of complexes is consistent with the aDP value of the tannin fraction estimated by the standard method (thiolysis).

Validation with Other Tannin Fractions. In order to validate these preliminary results, other tannin fractions were analyzed under this set of optimized conditions, that is, $1 \mathrm{mg} / \mathrm{mL}$ BSA concentration, 1:1 protein-to-tannin molar ratio, and trans-3, 5-dimethoxyhydroxycinnamic acid as matrix. All MALDI-TOF spectra were recorded on the diluted complex solutions except for the aDP 27.8 fraction, which required the nondiluted mixture. These experiments were carried out in duplicate.

All MALDI-TOF mass spectra showed a hump at higher $\mathrm{m} / \mathrm{z}$ values than the free BSA signal (Figure 6), with the precise shape of the hump varying from one spectrum to another. The signal extended over a larger mass range and its maximum was shifted toward higher $m / z$ values as the average degree of polymerization of the fraction increased. For each experiment, we calculated the mass difference between the top of the hump and the BSA signal. The molecular weights estimated in this way were compared with the values obtained from direct MALDI-TOF analysis and, for condensed tannins, to those determined by thiolysis (Table 3).

From these values, the average degree of polymerization (aDP) or the average number of galloyl units $(\mathrm{aDG})$ can also be calculated as

$$
\begin{aligned}
\mathrm{aDP}=(\mathrm{aMW}-2) / 288 \text { or } \mathrm{aDP}=(\mathrm{aMW}-2) /[288 \\
\\
(0.825)+440(0.175)] \text { for OPC } \\
\mathrm{aDG}=(\mathrm{aMW}-192) / 152
\end{aligned}
$$

Good reproducibility in replicate samples was obtained. The molecular weight values calculated from MALDI-TOF analysis of the BSA-tannin complexes were consistent with those estimated by thiolysis for the aDP 9.2 fraction but slightly higher than those estimated by other methods for the oligomeric fractions. For the largest polymers, however, MALDI-TOF data yielded much lower values (11.1 instead of the expected value of 27.8). These results suggest that this raw approach has limitations for aDP estimation and it does not readily convey information concerning the polydispersity of the fractions. 
Table 5. Mn, aDP, and PI Values Determined for Each Tannin Fraction from MALDI-TOF Analysis of the BSA-Tannin Complexes

\begin{tabular}{|c|c|c|c|c|c|c|c|c|}
\hline & \multirow{2}{*}{\multicolumn{2}{|c|}{ direct analysis of fractions }} & \multicolumn{6}{|c|}{ MALDI-TOF analysis of complexes ${ }^{a}$} \\
\hline & & & \multicolumn{2}{|c|}{$M_{\mathrm{n}}$} & \multicolumn{2}{|c|}{$\mathrm{aDP} / \mathrm{aDG}$} & \multicolumn{2}{|c|}{ PI } \\
\hline & av mol wt & $\mathrm{aDP} / \mathrm{aDG}$ & series 1 & series 2 & series 1 & series 2 & series 1 & series 2 \\
\hline Tara tannin & $860^{b}$ & 4.5 & 924 & 1038 & 4.8 & 5.6 & 1.07 & 1.14 \\
\hline $\mathrm{OPC}$ & $820^{c}$ & 2.6 & 1015 & 1161 & 3.2 & 3.7 & 1.13 & 1.13 \\
\hline DP 9.2 & $2652^{c}$ & 9.2 & 2650 & 2788 & 9.2 & 9.7 & 1.07 & 1.08 \\
\hline DP 27.8 & $8008^{c}$ & 27.8 & 8064 & 7811 & 28.0 & 27.0 & 1.18 & 1.17 \\
\hline
\end{tabular}

${ }^{a}$ Analysis of BSA-tannin complexes by mathematical processing. ${ }^{b}$ Direct analysis of the fraction by MALDI-TOF. ${ }^{c}$ Direct analysis of the fractions by thiolysis.
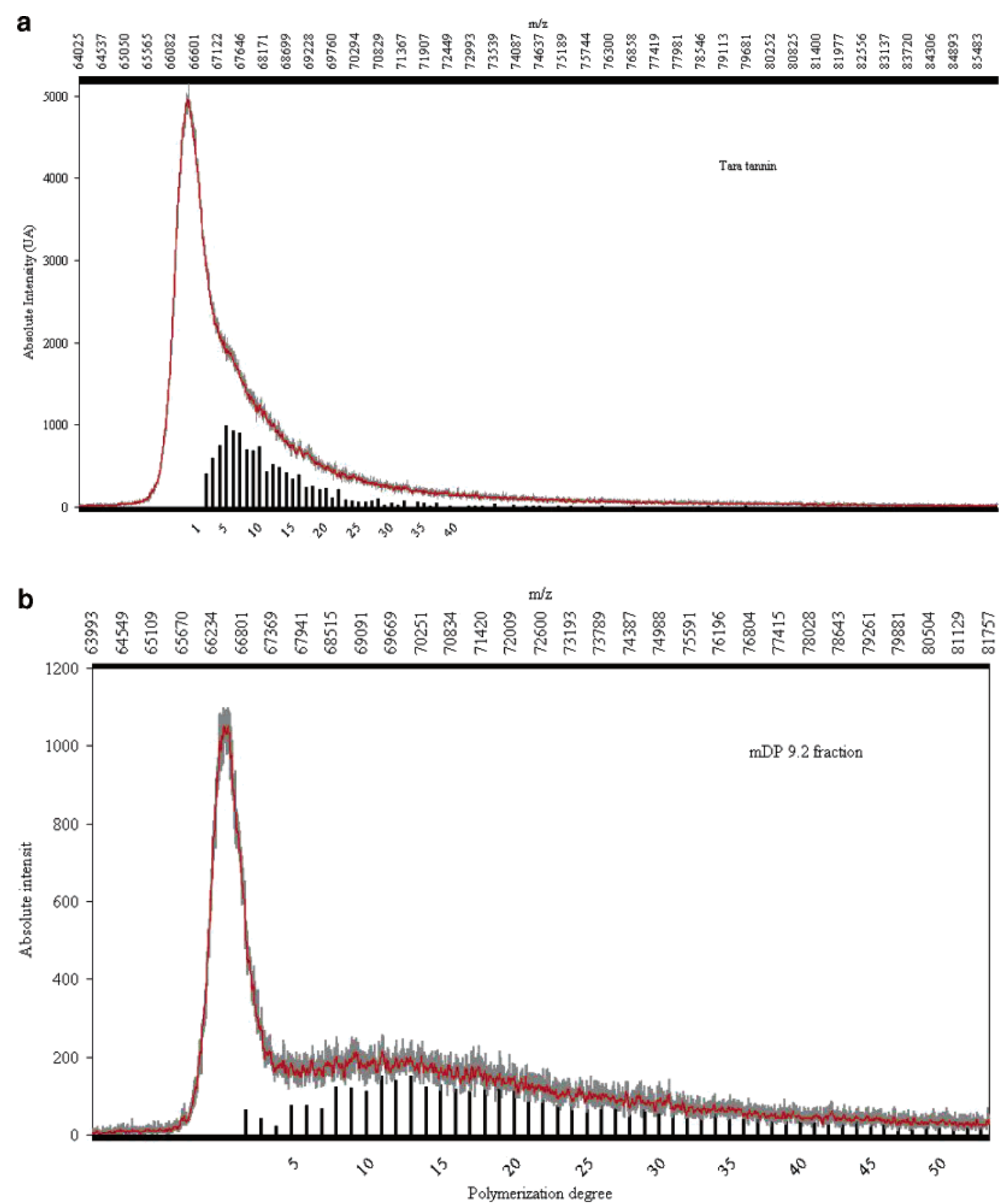

Figure 8. Raw spectrum (gray) and moving-average curve with background noise suppression (red) of BSA-tannin complexes and visualization of the size distribution of tannins (histogram) in the case of Tara tannin (a) and aDP 9.2 fraction (b).

Development of a Processing Method for aDP Calculation and Size Distribution Modeling. Polymer parameters, that is, the number-average molecular weight $\left(M_{\mathrm{n}}\right)$, weight-average mo- lecular weight $\left(\mathrm{M}_{\mathrm{w}}\right)$, and polydispersity index $(\mathrm{PI})$, can be calculated from the mass spectra of BSA-tannin complexes. In our case, the mass spectrum directly gives access to the polymer 
parameters of the BSA-tannin complexes, from which we deduce the corresponding parameters for the tannin fraction alone by substracting the $M_{\mathrm{n}}$ of BSA, assuming all signals correspond to 1:1 complexes. The first step of our processing was to select properly the mass range corresponding to signals of the complexes, which can differ from the mass range acquired. The starting point was arbitrarily selected at $m / z=61000$ for all experiments, whereas the end point was attributed to the $m / z$ value corresponding to the return of the raw signal back to the baseline for each experiment.

In order to eliminate, on the one hand, the variability of the solution spotting and, on the other hand, the variability of the mass signal-to-noise ratio, the moving-average (smoothed) curves were first established with every 30 points of the raw mass spectra of BSA and BSA-tannin complexes. This smoothing actually reduces the noise with minimal modification of the shape of the raw spectrum. However, to cancel out the varying baseline, the baseline at the beginning of the raw spectra (between 61000 and 65000 ), was subtracted over the entire mass range considered. The baseline actually corresponds to the minimum of the absolute intensities read in this part of the spectrum. For illustration, comparison of the raw and processed spectra obtained from the mixture of BSA and aDP 9.2 tannin fraction is shown in Figure 7. For each experiment, the mass spectrum was processed in this way, and $M_{\mathrm{n}}$ and $M_{\mathrm{w}}$ were determined for BSA and BSA-tannin complexes. The results obtained for the duplicate series are summarized in Table 4. The average molecular weight of the corresponding tannin fraction was then calculated by substracting the BSA $M_{\mathrm{n}}$ value from that of the complexes.

The $M_{\mathrm{n}}$ and aDP values calculated for apple condensed tannin fractions by this analysis of MALDI-TOF mass spectra are in good agreement with the values estimated by thiolysis (Table 5). Concerning OPC, results are higher than those obtained by thiolysis. The OPC fraction is probably contaminated by oxidation products of tannins formed either in the course of isolation or in storage. These oxidized tannins result from oxidative coupling of original tannins, leading thus to larger polymeric chains on average that may bind to BSA as compared to the nonoxidized ones. However, they are not actually taken into account in the thiolysis results because their biphenyl interflavan linkages are resistant to acidic cleavage, ${ }^{20}$ which may explain the discrepancy between MALDI-TOF and thiolysis results.

The values calculated from BSA-Tara tannin complexes are slightly higher than those obtained from direct MALDI-TOF analysis. This suggests that this direct method of analysis deviates somewhat from the actual composition of the gallotannin fraction, possibly due to the occurrence of fragmentation (cleavage of the galloyl ester linkages, releasing species of lower DG) or to the existence of complexes with higher stoichiometries than 1:1, as observed previously for another gallotannin, pentagolloylglucose. ${ }^{15}$ To better visualize the molecular weight distribution of each tannin fraction, a histogram can be built by extracting the discrete $m / z$ values of the complexes between BSA and the different tannin fractions, measuring their respective intensities, and substracting the contribution of BSA. The histograms of Tara tannin and aDP

(20) Guyot, S.; Vercauteren, J.; Cheynier, V. Phytochemistry 1996, 42, 12791288.
9.2 tannin fraction are displayed in Figure 8, showing the extent of galloylation (from 1 to 30 ) or polymerization (from 1 to 52 ).

\section{CONCLUSION}

MALDI-TOF analysis of BSA-tannin complexes is a novel method for determination of the higher molecular weight tannins that are poorly detected by direct mass spectrometry analysis, due to fragmentation and tannin aggregation or "stacking". The method has been applied and validated on condensed tannin fractions from aDP 2.6 through aDP 28, yielding $M_{\mathrm{n}}$ values consistent with those expected from classical thiolysis analysis. The new method represents a simple and rapid alternative to standard methods involving depolymerization in the presence of a nucleophilic agent that require HPLC separation and prior calibration of the reaction products for the determination of average molecular weight of polymeric tannin fractions. In addition to average molecular weight values, the method provides information on the polymer distribution parameters and thus can also rival time-consuming methods like SEC in this respect. The MS signals of the 1:1 BSA-tannin complexes were used for modeling the size distribution of tannin fractions. However, like SEC, the method suffers from the lack of suitable calibration solutions to validate the distribution parameters of polymeric fractions.

For gallotannins, reoptimization of the protein-to-tannin ratio between 10:1 and 1:1 could be tested since larger stoichiometry complexes seem to be favored in the case of small molecule targets such as hydrolyzable tannins.

A future development of the method reported herein would be to go further in the analysis of condensed tannins presumably involved in astringency perception. Thus, protocols based on tannin precipitation with BSA have been initially developed by Hagerman and Butler ${ }^{18}$ and further improved by Adams and Harbertson and co-workers ${ }^{21,22}$ for quantifying grape and wine tannins. However, these approaches cannot provide information on the nature of the precipitated tannins, while other studies have shown that larger molecular weight tannins are selectively precipitated out by proteins. ${ }^{23}$ Our approach might give further insights into the mass distribution of these precipitated tannins.

\section{ACKNOWLEDGMENT}

This work was financially supported by the Comité Interprofessionnel des Vins de Champagne (CIVC) and Moët \& Chandon Company. Thanks are due to Sylvain Guyot, who provided the tannin fractions from apple.

Received for review September 7, 2006. Accepted December 21, 2006.

AC061685+

(21) Harbertson, J. F.; Kennedy, J. A.; Adams, D. O. Am. J. Enol. Vitic. 2002 , $53,54-59$.

(22) Harbertson, J. F.; Picciotto, E. A.; Adams, D. O. Am. J. Enol. Vitic. 2003 $54,301-306$.

(23) Sarni-Manchado, P.; Deleris, A.; Avallone, S.; Cheynier, V.; Moutounet, M. Am. J. Enol. Vitic. 1999, 50, 81-86. 